\title{
Research on Visualization of Infographic Design under User Experience Thinking
}

\author{
Yi Zou ${ }^{1}$ and $\mathrm{Na} \mathrm{Qi}^{*}$ \\ ${ }^{1}$ School of Fine Arts and Design, Xihua University, Chengdu, Sichuan, 610039, China
}

\begin{abstract}
The visual design of the infographic is designed to compress complex information and present it to the audience through an intuitive and easy-to-understand expression, so that they can effectively absorb the content therein. With the continuous development of science and information visualization technology, the production methods and presentation forms of information charts have become more and more abundant, and the direction from two-dimensional information charts to multi-dimensional information charts and dynamic information charts has continuously evolved. This paper cuts in from the perspective of user experience, and proposes optimization suggestions for the current status of visual design of infographics.
\end{abstract}

\section{Introduction}

With the continuous development of computer technology, along with the multi-sensory reading needs and visual consumption requirements brought by the era of picture reading, the visual value of the design of information charts has continued to increase. As a type of communicative design in the data age, the production and presentation of information charts presents a multidimensional cross-fusion situation. From the initial flat image expression to the three-dimensional multidimensional dynamic display, from a single visual design to an intelligent visual design, information consumption is gradually formed. Information visualization is an important achievement in the field of computer graphics. Nowadays, with the emergence of rich information media and the establishment of open resource libraries for media platforms, the concept of information visibility derives new meaning from the continuous development of intelligent design and automation design, and further develops the meaning of communication from the meaning of information identification. In the age of scientific and technological innovation, the involvement of experience thinking can broaden the design visualization path of infographics and increase the visual value. Through people's multi-channel perception ability, it helps people to accurately, quickly and effectively understand the required information, enhances the audience's knowledge acquisition and emotional interactive experience, and can also cultivate and enhance the audience's design thinking and aesthetic ability.

\section{Design visualization for infographic}

\subsection{Definition}

Infographic design is included in the large category of information design and is part of design visualization. Infographic design visualizations can be as simple as information visualization. An Yaoshun and He Jie believe that infographic design is to guide and help people to interpret information more quickly, deeply and effectively by visualizing the information and making full use of the processing power of human visual system[1]. Cai Shunxing believes that the design visualization of information charts aims to use computer technology to refine, extract, transform and integrate the characteristics of information objects, and to use graphics, images, symbols and other visual forms to highlight the content characteristics and related semantics of information[2]. The core of information visualization design is that designers use appropriate methods to represent complex information and data, and comb information through visual symbols such as graphics, text, layout and color, and even combining dynamic images, the potential relationship between data is represented in a more visual way[3].

Infographic is an information tool[4]. The early infographic design only had the concept of a simple data set. Up to now, modern information graphic design has been influenced by interdisciplinary theories such as psychology, statistics, sociology, etc., and has continuously improved the ability of visual expression, the artistic expression of visual elements is valued[5], and the concept of making infographics has changed from making content to making products, using technology to achieve a good reading experience and customized services[6].

\subsection{Common categories}

*Corresponding author's e-mail: happylife8183@163.com 
The production types and content of information charts are diverse. If the content is divided, the boundaries are relatively vague and it is difficult to generalize. This paper has roughly divided the categories in the form of information charts, which are two-dimensional infographic, multi-dimensional infographic, and dynamic infographic, and subdivide them into categories.

2.2.1 Two-dimensional infographics. Two-dimensional infographic can be roughly divided into text-oriented, image-oriented, chart-oriented, and indicator symbols, and most of them can be designed by cross-fusion. Most of these categories are mainly flat displays, of which typographic layout is the core design element. Static images are the most commonly used form of expression in the information visualization environment[7], most twodimensional information charts use static images.

The text-oriented category mainly uses large-length text as the main display content, which is most common in news infographics. The image-oriented category is mainly based on the core image as the design benchmark. The main types of charts and indicators are to clarify the relationship between various elements, build the information structure of the product, and form the final visual logic. As shown in Figure 1, the picture on the left is the Lisbon metro map, and the picture on the right is the infographic work 'Burger' by Korean designer Sunghwan Jang. Both of the above charts are two-dimensional infographics.
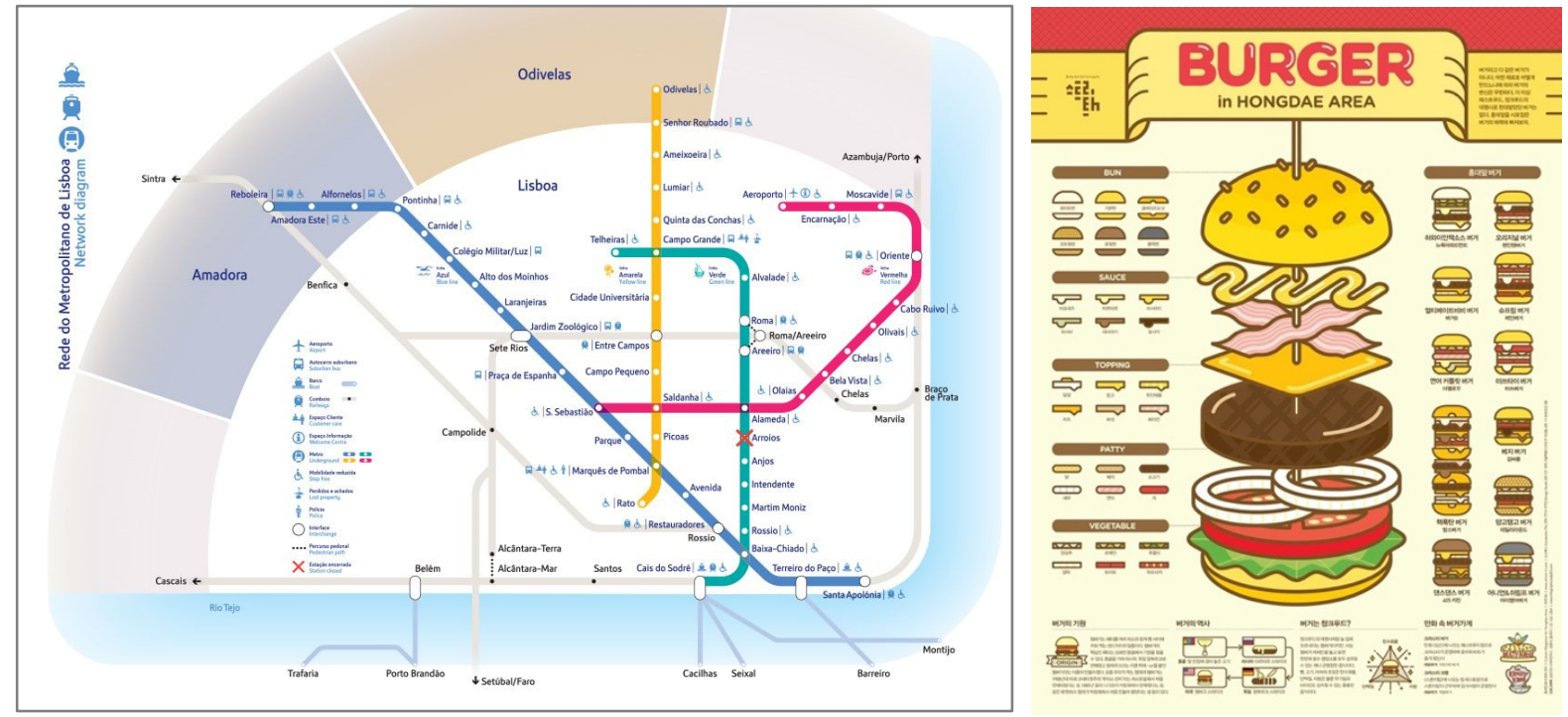

Figure 1. Two-dimensional infographic.

2.2.2 Multi-dimensional infographics. The multidimensional infographic is designed to simulate the threedimensional virtual situation by using the spatial and temporal dimensions, explain and split the information from a multi-dimensional perspective, sort out the changes at various levels and reveal the relationships among them. It is easy to create a sense of spatial structure, and provides new perspective on the interpretation and interpretation of information. As shown in Figure 2, the scene display map of the XGEOMATICS intelligent experience area visualizes multiple spatial scenes and physical parameters, condenses and refines complex content, and models a large number of text descriptions or tedious structures. The screen layout design presents multi-dimensional scene information, which enriches the infographic. 


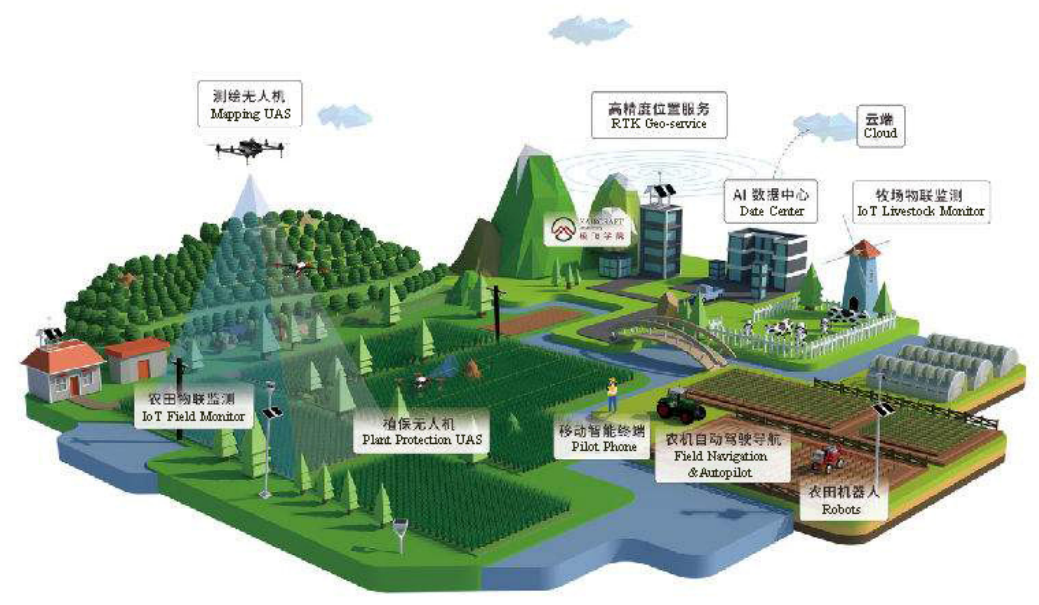

Figure 2. XGEOMATICS intelligent experience zone scene display map.

2.2.3 Dynamic infographics. With the continuous indepth reform and development of visualization technology and digital media, the presentation and production methods of information charts have become more intelligent and diverse, and added new tool forms that demonstrate the interactivity, fun, and creativity of dynamic infographics. The dynamic interaction trend is due to the continuous development of online media and the advent of the smart economy. Users are increasingly relying on smart devices and are more actively using new media platforms to obtain content information. Intelligent technology has gradually penetrated into the new media industry.

Figure 3 is a screenshot of a popular science video made from the media account 'PaperClip'. The producer presents the process and data of the train operation control system in a visual form. Such dynamic information charts have a great guiding role, breaking a single visual expression, and awakening the user's multi-channel senses such as hearing, perception, touch, and vision. Give users high-quality content products, help users achieve a good reading experience, establish a good visual aesthetics, and greatly enhance the user's emotional experience.
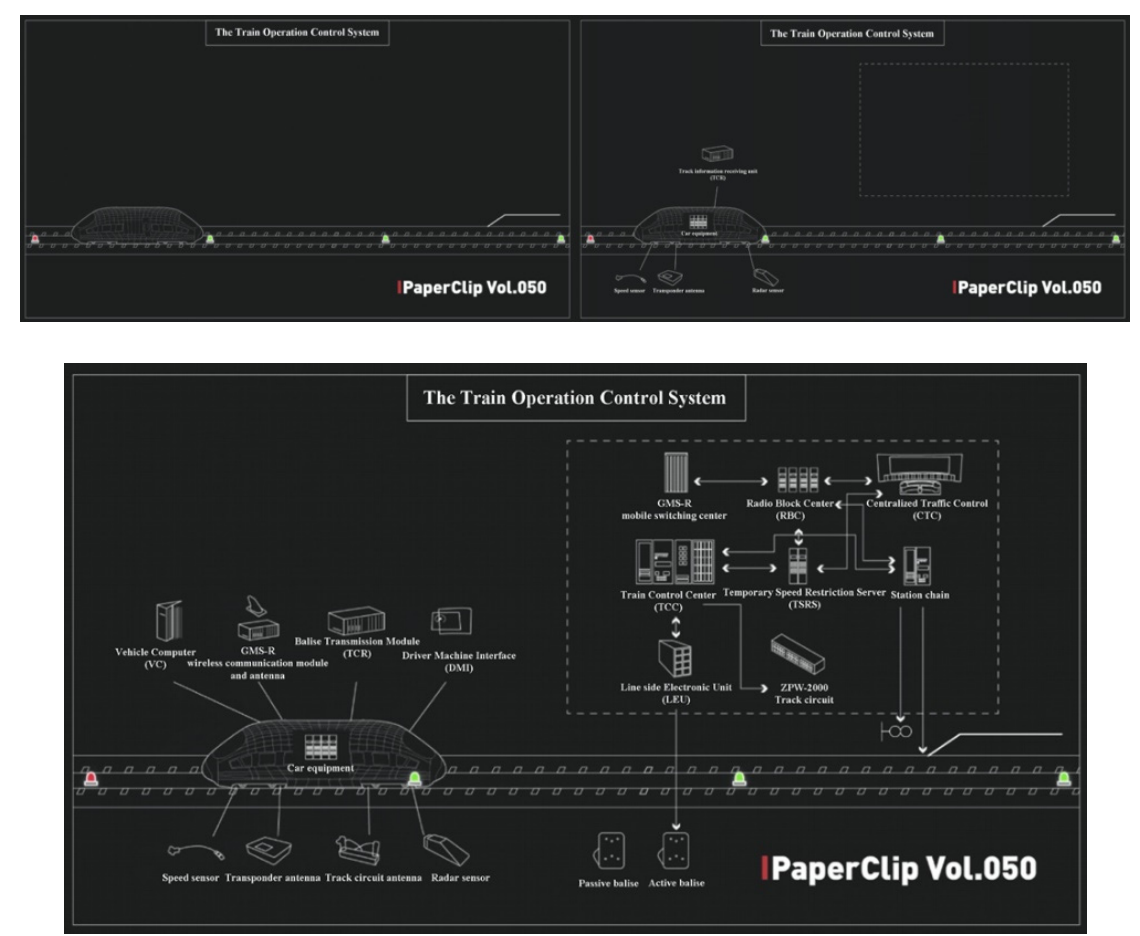

Figure 3. The train operation control system. 


\subsection{Development trend}

2.3.1 Multi-dimensional cross fusion. The design visualization path of infographics is becoming more and more media-rich, integrating video, audio, dynamic interaction, multi-dimensional display, etc., and combining dynamic and static, physical and virtual. With the continuous development of science and technology, the visualization of information design will inevitably move in the direction of multi-dimensional cross fusion. In the visual design of infographics, the operability of technology should be fully analyzed, and the richness of media should be used to explore the possibility of new media, new technology, and new forms [8]. The mode of communication and the carrier of communication provide convenience for the dissemination of information. The technical level will inevitably play an important role in the visual production of the infographics. The multidimensional cross means in the infographic enhances the richness of the dissemination content, so that the audience can achieve effective information interpretation and visual aesthetics when browsing the infographic.

2.3.2 Visualization of intangible concepts. In the digital media era, the visualization of information design includes more content. In addition to the visualization of data and text content, the visualization of intangible concepts has also followed, such as the visualization of sound, the visualization of smell, and the visualization of emotion. With the deepening of user needs and the evolution of visual technology, people are constantly trying to visualize various types of information and abstract thinking. As shown in Figure 4, Daniele Quercia et al. used visualization technology to transform olfactory information into a panoramic street odor visual map. Figure 5, British artist Kate Mclean recorded the scent in detail using different colors and various irregular ring patterns to visualize the range of scent. The development of science and the continuous improvement of visualization technology means, the visualization and rationalization of intangible concepts have created a fresh visual experience for the audience, and put forward new requirements for design visualization.

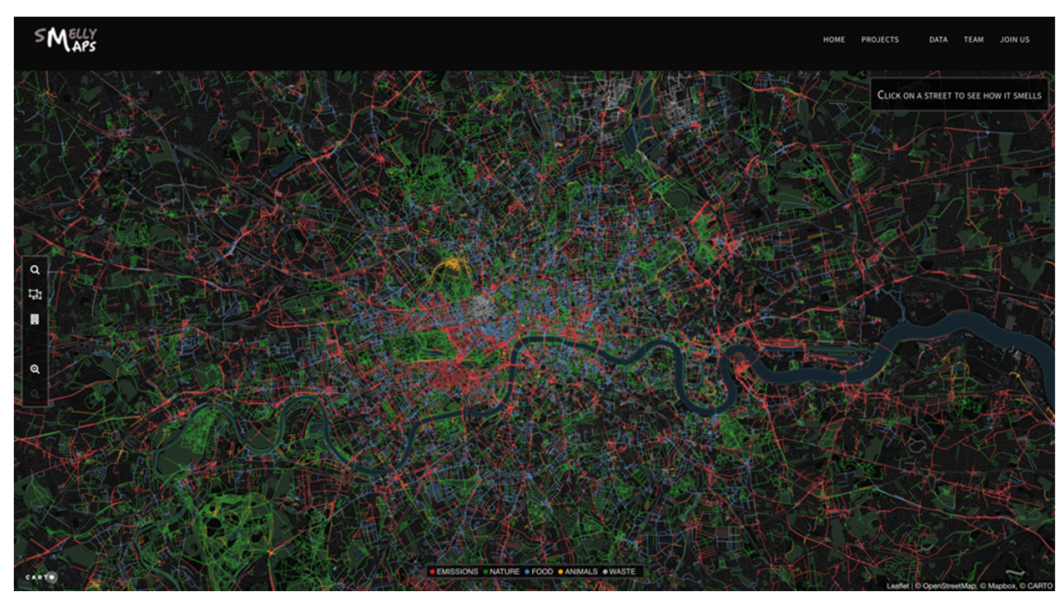

Figure 4. London smelly map.

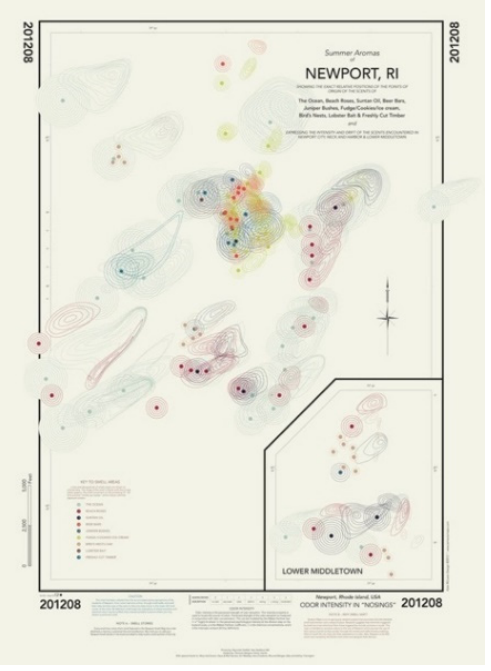

Figure 5. Newport smelly map. 


\section{Visualization process}

Information visualization includes data visualization, information graphics, knowledge visualization, scientific visualization, etc[9]. The visualization process is based on the extraction, transformation, mapping, high abstraction and integration of the information object's feature, and the use of graphics, images, animations and other means to represent the content characteristics and semantics of the information object[10]. As shown in Figure 6, the visualization process is a process of forming a new visual paradigm after outputting the aesthetic logic of information.

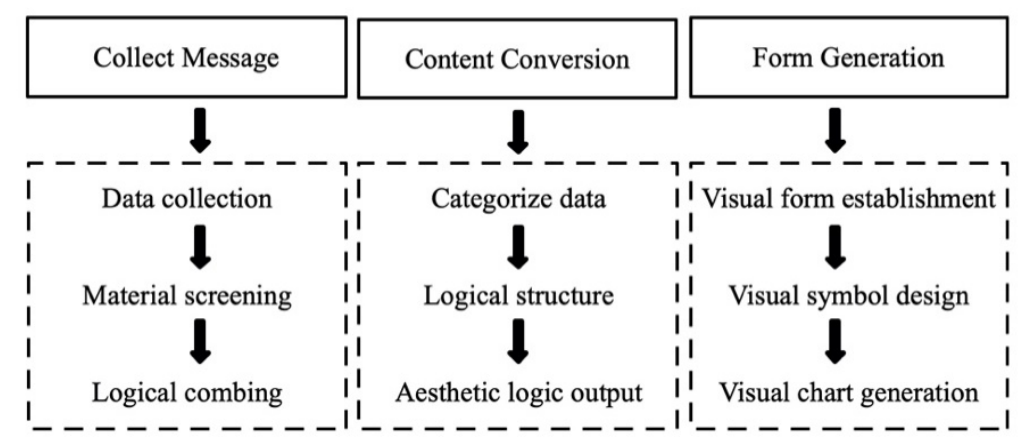

Figure 6. Visualization process.

\subsection{Collect message}

The process of infographic design and visualization is the process of generating content products, the core of which is to convey accurate and effective information to the audience. It is a logical combing and derivation process. After determining the content and theme, the first step is to collect and filter the material. This step is the filtering process of the original material and related data. It summarizes a large amount of text information and sorts out the text logic, to determine the accuracy and objectivity to build text information structure.

\subsection{Content conversion}

Content conversion is a necessary process for the transformation from text logic to visual logic. The focus is on how to extract a large amount of text information to form a concise visual language and complete the attribute conversion between different content. First of all, we should classify and distinguish data, then set up a logical structure of information, clarify the relationship between materials and data, and find appropriate expressions. We will analyze and convert text information, statistical data, and related abstract concepts into visual images to complete the output of aesthetic logic. This reduces the cognitive load of the audience.

Refining and transforming content is the designer's transformation of text content into appropriate schemas through intellectual cultural interpretation, unique creative perspectives, and accurate aesthetic reconstruction[11]. The designer comprehensively displays the content of different attributes in the same space, embellishes it into various image styles or dynamic displays that are easy to be understood and read by the broad audience, and gives it aesthetic value and discerning meaning, thereby completing text logic to visual logic conversion.

\subsection{Form generation}

Form generation is the process of visual modification of text to graphics or dynamic images. Firstly, the visual form, that is, the overall visual presentation of the chart, needs to be determined. Second, the visual symbols that match it must be selected, which must conform to the visual context of the infographic. Finally, the design visualization of the infographic is generated. The visual representation and construction of information charts should be a process of gradually establishing the correlation between data and "real". This process is not a purely technical proposition, nor is it a visualization of the design category, but a mining and interpretation of the social nature of data[12]. The generation of the information chart contains the designer's independent thinking on how the data information is visible, clarifies the context of the text for the user, and finally generates the information chart to be disseminated in a visual form.

\section{User experience thinking involved in infographic design}

The design of infographics is not only the visualization of information content, but also an important way to spread social content and promote products and services. Graphic design in the context of information visualization has spawned a variety of expressions and production methods, the means and carriers of information graphics have also changed, and designers have begun to involved in user experience thinking when visually designing infographic, in order to improve audience reading efficiency and user experience when browsing and understanding infographics.

\subsection{Leverage smart interactive trends}

In accordance with the development of science and technology and the era, actively using technology and 
smart tools can help shape the scene-oriented information space and discover new aesthetic experiences of infographics. Through the linkage of vision and perception, the substantive meaning of information content and visual visibility are connected to create a matching interactive environment for the public and form a good interactive experience, thereby promoting the formation of the interactive value of information, media and audience .

Dynamic and interactive infographics pay more attention to the interactive relationship between participants and content display, accompanied by natural participatory interaction behavior. The designer first selects a suitable visual design solution from the user's perspective, which satisfies the reasonable connection between text logic and visual logic. Good information image design and visual narrative logic deliver more complete and accurate visual content to the audience through dynamic display. With the help of new media communication vehicles and intelligent interactive tools, it can provide fresh ideas for the design and visualization of infographics. Through the change of visual forms, it integrates the multi-dimensional requirements of content products, and grafts and replaces the different attributes of two-dimensional planes and three-dimensional spaces.

\subsection{Creating interactive experience situations}

The continuous development of new media technology has brought about a new aesthetic turn, which has enabled people to expand and surpass the traditional art aesthetics and display forms. It has new aesthetic qualities such as aesthetic narrative graphics, pluralistic aesthetic elements, aesthetic subject interactivity, and aesthetic presentation technology. It has changed and overturned the production mode and aesthetic paradigm of infographics in a sense[13]. The presentation form of infographics is developing towards rich media. Sensory experience, interactive experience, and emotional experience have become several positive evaluation indicators of the audience's satisfaction with the infographic. The use of symbols related to human emotion in visual language, incorporating artistic aesthetic and emotional experience, can stimulate the creative interactive experience between picture readers and designers[14]. For example, customize the visual presentation form for personal aesthetic preferences. It can greatly mobilize personal emotional experience, and improve user sensory experience.

The group interactive experience can enable people to have a great sense of joy and participation. The user actively participates in the interaction of information content, and proposes new content insights and content judgments to help the optimization and improvement of information charts. It is a feedback mechanism based on good interaction.

\section{Conclusions}

The visual design of the infographic is a content visual logic framework formed by the designer injecting design thinking and emotional expression. After the text is gathered, it is reshaped to form an image. In this process, the rapid combination of information logic and visual logic is achieved, which triggers the audience's multichannel perception, mining the value behind the information presentation results, and constructing a visual context for the design of the infographic.

Users' needs for information have always existed. The richness and advanced nature of digital media have led to the rapid development of infographic presentation methods and production technologies, giving audiences a new sensory experience, broadening the value of infographic spread, helping to build knowledge identity, enhancing the knowledge visualization experience, and forming a good interactive experience and emotional cognition. Visualization of the design of infographics from the perspective of user experience is not only a single process of transmitting visual information forms, but also an output of the value of information aesthetics. While gaining a good reading experience, the audience can cultivate and enrich their design thinking ability and deeply understand the social diversified information to achieve accurate information structure and good information vision symbiosis. Therefore, making full use of the richness of visual technology and media and the convenience of the platform, and taking the good user experience as the starting point, can enhance the fun, creativity and visual value of the infographics, and promote the realization of efficient information transmission and artistic aesthetic functions achieve.

\section{References}

1. AN, Y.S., He J. (2018) Visual Representation of Product Infographics in Sales Packaging Design. Packaging Engineering, 39(02):48-54.

2. CAI, S.X. (2019) Purification, Expression, Order, and Pleasure: on the Conceptual Dimensions of the Infographic Design for Scientific Journals. Chinese Journal of Scientific and Technical Periodicals, 30(04):336-342.

3. LI, R.Y. (2016) Information Visualization Design Method in Electronic Publication. Technology and Publishing, (05):86-89.

4. Hieoyuki, K., WU, X.F., GU, Y. (2013) Graphic Force: Learn from Top Designers to Make Infographics. Post Telecom Press, Beijing.

5. HAO, N.H. (2016) Infographics Design in the Context of Information Design. Packaging Engineering, 37(18):76-81.

6. XU, X.D. (2018) Inheritance and Evolution of Infographics in the New Communication Environment. Contemporary Communication, (01):102-105.

7. YANG, X. (2016) The Basis of Selection and Judgment of Visual Representation Form of Static Images and Animation of Information Visualization. Art and Design, (11):121-123.

8. XU, J., SUN, X.M. (2017) Process Visualization Teaching Practice Based on International Teaching Exchange. Art and Design, (05):105-107. 
9. BAIDU. (2016) Information visualization. http://baike.baidu.com/link?url=WR0mmeK5BVqib z02qt3FNLyC8c_D3z9QD00_VuT5yYK7Z WV5UGgIwzdSy06A41dlxUV2vLrbp7onpkrjnR2K.

10. HUANG, Y.K. (2015) Application of Information Design Based on Visualization in Newspaper Editing. China Publishing, (01):45-48.

11. CAI, S.X. (2019) Purification, Expression, Order, and Pleasure: on the Conceptual Dimensions of the Infographic Design for Scientific Journals. Chinese Journal of Scientific and Technical Periodicals, 30(04):336-342.

12. LIAO, H.Y. (2016) "Image" in "Image": The Visual Representation and Construction of Information Graphic. Journal of Central South University(Social Sciences), 22(01):208-213.

13. ZHANG, Y. (2015) The Analysis of Image Narration and Aesthetic Changes in Data Journalism. Friends of Editors, (03):85-88.

14. OUYANG, M.Y., XIAO, Q., GAO, J.S. (2017) Creative Design of Map in the Context of Information Visualization. Packaging Engineering, 38(24):61-65. 